\title{
The research of new shunt hybrid active power filter
}

\author{
Hongying Deng ${ }^{1}$, Tingjian Zhong ${ }^{2}$ \\ Jiangxi Vocational and Technical College of electricity, Nanchang ,330032 China \\ ('jxdlztj@163.com, ${ }^{2}$ reddhy@163.com)
}

\begin{abstract}
Keywords: power grid quality, harmonic, power filter, simulation, suppression
Abstract: the harmonic components is the additional waveform in the power grid, the harmonic loss can directly reflect the quality of the power grid, the harm of harmonic influences to the power grid and the safety of the equipment, in this paper it will be introduced a new type of harmonic suppression measures, through the analysis of the working principle and structure of shunt hybrid active power filter, the electrical principle model and equivalent circuit are deduced. Through the design of the simulation parameters, it maps the harmonic suppression of waveform before and after, and also it is concluded that the new shunt hybrid active power filter should be well restrain harmonic components, so it is important practical significance to the entire power grid safety and quality.
\end{abstract}

\section{The introduction}

With the development of power electronic technology and the use of equipment, a large number of harmonics will pour into the power grid, and result it is caused the entire power system harmonic pollution and asymmetry, caused by the harmonic of fault and accident occur repeatedly. The harmonic loss greatly reduces the efficiency of power generation, transmission and power equipment ${ }^{[1]}$. Increasing the copper loss of transformer, resulting in rise of temperature rise and noise generation; it also affects the additional loss of other power equipment and the working accuracy of electronic equipment and shorten the life of electrical equipment. It also interferes with the normal work of other communication systems. Therefore, it is necessary to take effective restraining measures with power grid harmonic.

Traditional reactive power compensation and filtering methods already can not adapt to the user demand for the power quality, a new type of shunt hybrid active power filter is a kind of active filter and passive filter series, and then shunt to the power grid and nonlinear loads, it combines the advantages of passive filter and active power filter, and the same time it can realize with smaller active harmonic compensation device to capacity of large capacity, and can realize high pressure system of harmonic compensation and base wave reactive power compensation, so it can well realize the control of harmonic pollution and improve power quality, and also it has wide application in the whole power system and has well development trend.

\section{Work principle and system structure diagram}

Shunt hybrid active power filter (HAPF) system structures are composed of main circuit and the additional circuit, HAPF main circuit has the active filter APF and passive filter PF by coupling transformer $\mathrm{T}$ together ${ }^{[5]}$; The additional circuit is composed of control circuit, drive circuit, DSP processor and so on, as shown in figure 1. In the main circuit the PF consists of three, five, seven times of three groups of single tuned filter and high pass filter, its main purpose is used to filter out three, five, seven times harmonics and higher harmonics, also it greatly reduces the capacity of APF. The main purpose of APF is to filter the partial harmonics of the system, improve the filter characteristics of $\mathrm{PF}$, and also suppress the whole system resonance. Coupling transformer $\mathrm{T}$ is used to isolate electrical between the APF and PF, and also it is facilitate to the maintenance of the inverter, safe and reliable, and at the same time changing a larger parameter can reduce the capacity of APF, can significantly reduce the investment of the whole system. HAPF additional circuit in the control circuit is through the sensor to detect the grid voltage and current, follow detect the harmonic current ,gaining the reference voltage of APF, through the A/D conversion, come into the 
PWM pulse in which generates relevant switch by the driving circuit is sent to the main circuit of APF, realizing the thyristor conduction, finally it will produce amplifier offset current in order to realize the compensation function to the load, so as to getting the ideal power supply current, achieving the effect to suppress harmonic wave.

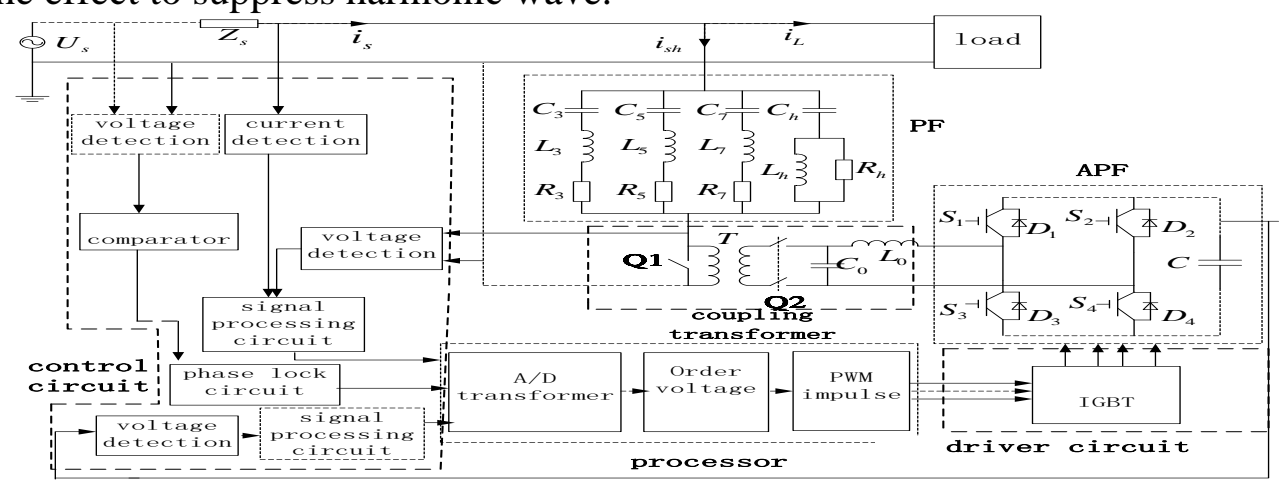

Figure 1 system structure diagram

\section{Electrical model and equivalent circuit diagram}

Before analysis of the HAPF filtering principle and characteristic ${ }^{[4]}$, it needs to simplify the hybrid power filter, concluded that the equivalent circuit diagram, now as single HAPF for example, it looks load as a source of harmonic current, the equivalent to current is $I_{S h}$, the equivalent impedance of $\mathrm{PF}$ is $Z_{P F}$, we can put $\mathrm{APF}$ as a voltage source, the equivalent voltage of APF is $U_{A P F}, \mathrm{~T}$ can be as the ideal coupling transformer, its change ratio is $\mathrm{n}: 1$, so we can get the electric model of HAPF, as shown in figure 2 .

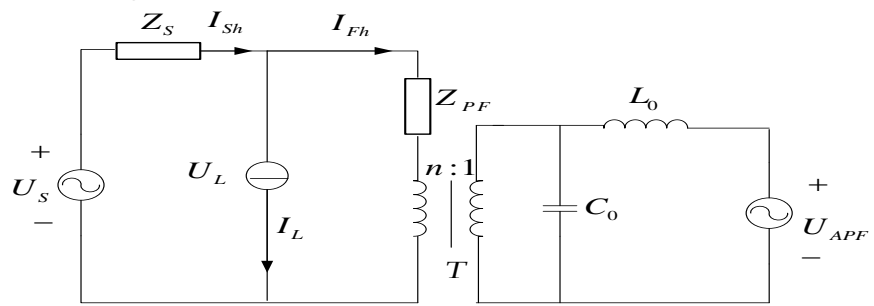

Figure 2 single phase HAPF electrical model

By converting the APF and output filter to the coupling transformer network side, so the equivalent circuit diagram of the single phase HAPF electric model is obtained, as shown in figure 3.

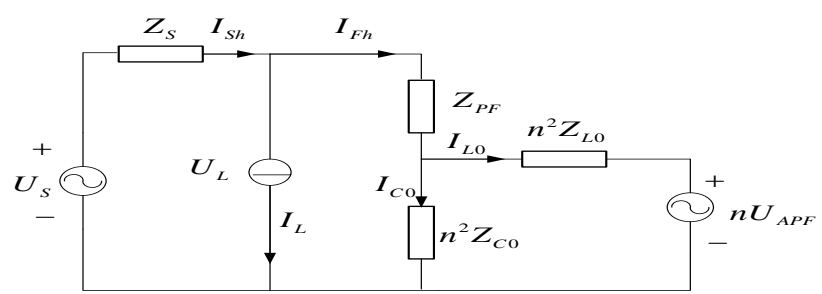

Figure 3 single phase HAPF electrical model in network side

Following the Circuit theorem, the equivalent circuit is shown in figure 4 . in expressions:

$$
K_{1}=\frac{n^{2} Z_{C 0}}{n^{2} Z_{C 0}+n^{2} Z_{L 0}}=\frac{Z_{C 0}}{Z_{C 0}+Z_{L 0}} \quad \text { (1) } \quad K_{2}=\frac{n^{2} \cdot Z_{L O} \cdot Z_{C O}}{\left(Z_{L O}+Z_{C O}\right)}
$$




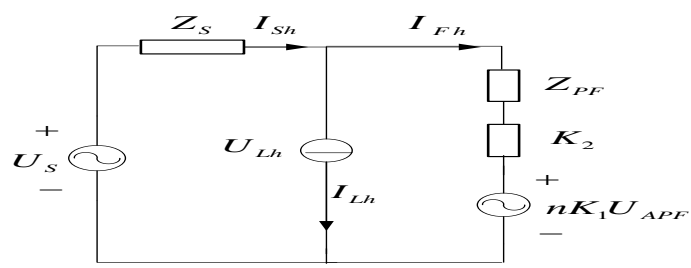

Figure 4 single phase HAPF equivalent circuit in network side

It can be seen from the figure above, there are three power sources that affect the harmonic current $I_{S h}$, Namely they are the power grid side voltage $U_{S h}$, inductance $I_{L h}$ and APF filter $^{n K_{1} U_{A P F}}$, so it can be obtained from the system harmonic current $I_{S h}$ according to the superposition theorem.

$I_{S h}=\frac{1}{K_{2}+Z_{S}+Z_{P F}} U_{S h}+\frac{K_{2}+Z_{P F}}{K_{2}+Z_{S}+Z_{P F}} I_{L h}-\frac{n K_{1}}{\left(K_{2}+Z_{S}+Z_{P F}\right)} U_{A P F}$

It can be obtained from the formula in order to make the harmonic current of the power grid small or zero, it can be controlled $U_{A P F}$ to improve the effect of load filtering.

\section{Parameter design and simulation model}

This article is by MATLAB7.5 /SIMULINK to establish a power supply system of the hybrid active power filter (HAPH) as a simulation model, now we go ahead respectively parameter design on passive filter and active filter, follow the program debugging, analysis and simulation ${ }^{[4]}$, we get the parameters of the passive filter as shown in table 1.

Table 1 the parameters of the passive filter

\begin{tabular}{lccc}
\hline Filter parameters & $\mathrm{C}\left(\mu_{\mathrm{F}}\right)$ & $\mathrm{L}\left({ }^{m} \mathrm{H}\right)$ & $\mathrm{R}(\Omega)$ \\
\hline 3 times single tuned filter & 18.8 & 65.0 & 1.5 \\
\hline 5 times single tuned filter & 9.2 & 40.7 & 1.9 \\
\hline 7 times single tuned filter & 9.3 & 23.5 & 1.6 \\
\hline high pass filter & 9.5 & 7.1 & 35.8 \\
\hline
\end{tabular}

Active filter APF ac side output the inductance $\mathrm{L}$ is $0.0076 \mathrm{H}, \mathrm{DC}$ side capacitance $\mathrm{C}$ is $0.004 \mathrm{~F}$, the coupling transformer ratio $\mathrm{n}$ is 9 , triangle wave modulation frequency select $20 \mathrm{KHZ}$. The single phase HAPF simulation model is obtained through the design of parameters, as shown in figure 5.

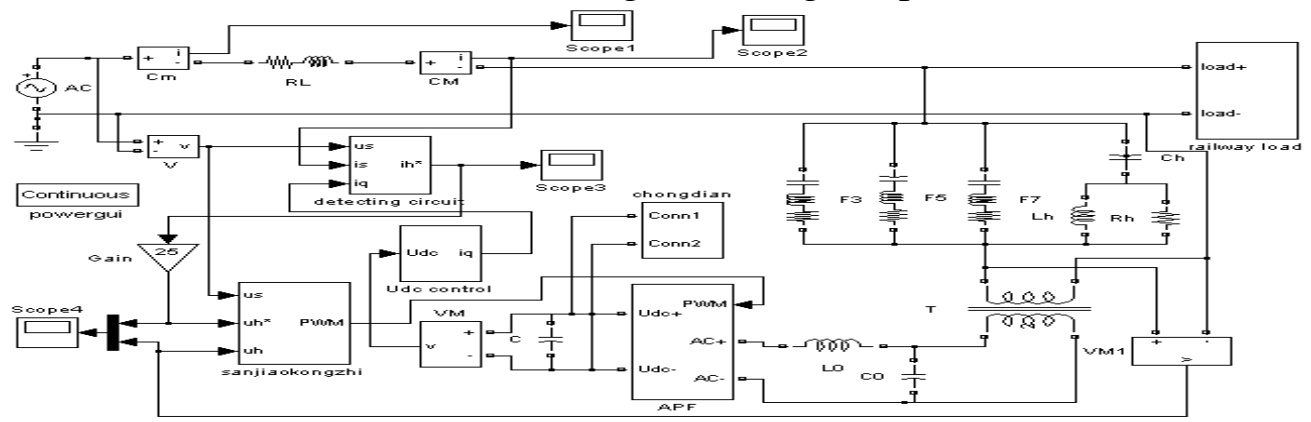

Figure 5 single phase HAPF simulation model

\section{Waveform analysis and expected results}

It can be seen from the simulation results, follow inputting shunt hybrid active power filter (HAPF) after compensation, as shown in figure 6and 7, the load current harmonic component is greatly reduced, almost it closes to the sine fundamental component, Moreover, the THD of the system power current is reduced to $2.31 \%$ from $21.26 \%$ before the compensation, while the current and voltage are in the same phase, compensating for the reactive power, improving the power factor and improving the working efficiency of the whole grid. 


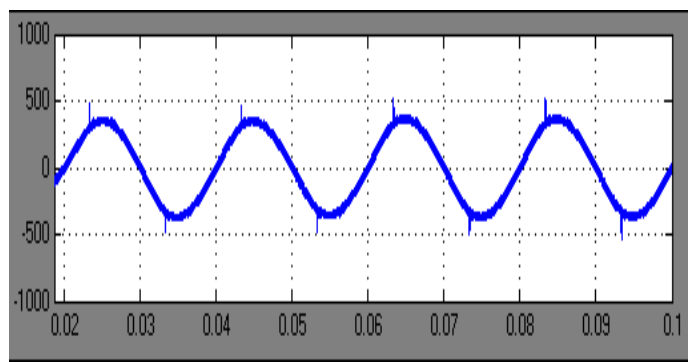

Figure 6 input the network side waveform of HAPF

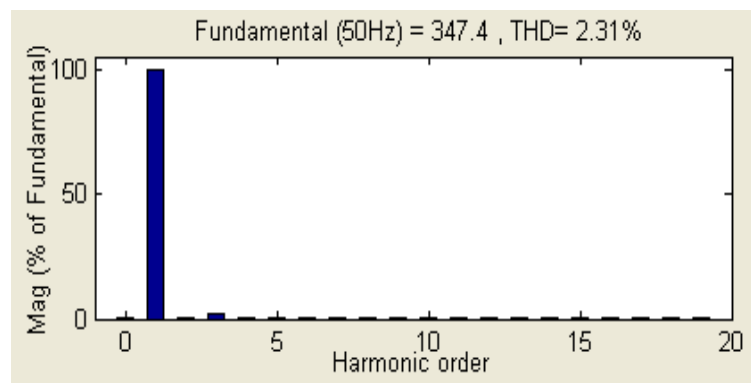

Figure 7 input the network side current spectrum of HAPF current

\section{Conclusion}

In this paper, at first we analyze the generation and harm of harmonics in the power grid, so we put forward a new type of harmonic suppression measures, based on the analysis of its working principle and structure, deduced the electrical principle of the model and equivalent circuit, through the parameter setting, building a simulation model of single-phase shunt hybrid active power filter, following the simulation results and waveforms shows, using the shunt hybrid active power filter can effectively compensate for the harmonic current, so it has the important practical significance to the whole power grid safety and quality.

\section{References}

[1]Hu huihui. Research of shunt hybrid active power filter $\quad[\mathrm{J}]$. Journal of Shanghai electric power institute, 2015.31 .

[2]Luo an. High-capacity hybrid power filter for harmonic suppression and reactive power compensation in the power substation [J]. Proceedings of the CSEE, 2004.24.

[3] Wenjin Dai,Taiyang Huang,Na Lin. Single-phase Shunt Hybrid Active Power Filter Based on ANN. Fourth International Conference on Fuzzy Systems and Knowledge Discovery, Haikou,24-27 August,2007

[4] Dai Wenjin, Huang Taiyang. Design of Single-phase Shunt Active Power Filter Based on ANN.2007 IEEE International Symposium on Industrial Electronics,Spain,June 4-7,2007-11-7. [5] L.H.Tey, P.L.So and Y.C.Chu. Neural network-controlled unified power quality conditioner for system harmonics compensation. Proceedings of the IEEE/PES Transmission and Distribution Conference and Exhibition Asia Pacific, Japan. 2002. 